\title{
File (in ordine sparso)
}

\section{Lines (randomly)}

Giovanni Lodi

Dipartimento di Scienze Biomediche, Chirurgiche e Odontoiatriche

Università degli Studi di Milano

via Beldiletto 1/3 Milano 20142

Italia

giovanni.lodi@unimi.it

Sabato ho fatto la coda per andare a comprare i giornali, sarei poi dovuto andare al supermercato dall'altra parte della strada, ma c'era una fila di solitari mascherati in attesa di entrare. Allora ho deviato verso il negozio di surgelati, dove ho aspettato solo una decina di minuti.

"Fare la fila ai negozi". Alla mia generazione non era mai capitato; al massimo ne aveva sentito parlare durante la guerra fredda, a proposito di paesi mai visti, dove i beni di consumo erano scarsi. Per le generazioni che precedono la mia, penso invece che sia un'espressione che riporta alla guerra. Adesso ogni generazione ha la sua fila.

Donne e uomini che oggi fanno il loro lavoro perché "prima c'è chi ha bisogno" e non "prima ci sono io". leri erano buonisti, oggi sono eroi, angeli. Piroette semantiche poco interessanti. Più importante è sapere che molti tra loro, troppi, sono precari e mal pagati. Sono sicuro che finita questa cosa, preferiranno un lavoro sicuro e uno salario adeguato, piuttosto che mettersi in fila per una bella medaglia di parole (che costa pochissimo). Staremo a vedere.

Non so in che fase saremo quando leggerete questo editoriale, ma non esiste un momento sbagliato per dire grazie. Se però dovessi mettere in fila tutte le persone che in questo momento si meritano un mio grazie, altro che supermercato. Quindi mi limito a tre grazie. Alle amiche e agli amici con cui lavoro in ospedale (molti precari e malpagati), perché senza di loro i pazienti che dovevano iniziare le cure oncologiche, quelli con importanti malattie delle mucose e quelli che richiedevano trattamenti urgenti, non sarebbero stati accolti e curati, come invece è successo. Ai colleghi che dal tutto il mondo mi hanno scritto preoccupati, perché mi hanno insegnato quanto la solidarietà faccia bene. Non lo sapevo. Alle donne di EDRA, perché hanno permesso che anche questo numero di Dental Cadmos vi arrivasse tra le mani.

Anzi, ci metto anche un grazie ai no-vax, tutti. Quelli che, disponibile il vaccino, si metteranno pazientemente in fila ad aspettare il loro turno e quelli che, irriducibili, renderanno più breve l'attesa per tutti noi.

Buona lettura, 